\title{
Deutsch in Europa - sprachpolitisch, grammatisch, methodisch
}

Dieser Band thematisiert das Deutsche in Europa aus drei Perspektiven: sprachpolitisch, grammatisch und methodisch. Die deutsche Sprache hat sich innerhalb Europas als Teil einer europäischen Sprachengemeinschaft entwickelt. Heute besteht ein ständiger Austausch zwischen diesen Sprachen; die politischen Rahmenbedingungen in der Europäischen Union und darüber hinaus werfen konkrete sprach- und bildungspolitische Fragen auf. Große Bedeutung besitzen Themen wie der Umgang mit Sprachenvielfalt, der Mehrsprachigkeit in Deutschland und Europa oder der Erwerb des Deutschen vor dem Hintergrund unterschiedlicher Voraussetzungen der Lernenden. Von besonderem wissenschaftlichen Interesse ist die Frage, wie sich Sprachen zueinander unter diesen Bedingungen beeinflussen und in welcher Weise sie sich langfristig verändern.

Im Kontext einer solchen Sichtweise des Deutschen in Europa sind Ansätze zu einer sogenannten Eurolinguistik oder zum Konzept eines europäischen Sprachbundes entstanden. Welche Rolle spielt die deutsche Sprache in derartigen Modellen, welche Konvergenzen und Divergenzen sind im Vergleich zu anderen Sprachen Europas festzustellen und wie wirkt sich dies auf die Deutung der Dynamik des Wandels aus? Derartige Fragen berühren unmittelbar auch die Dimension der methodischen Zugänge und der in der Forschung verwendeten Sprachressourcen. Darüber hinaus ist das Thema für Lehre und Forschung in der internationalen germanistischen Linguistik von großem Interesse, insbesondere in Hinsicht auf sprachvergleichende oder -kontrastierende linguistische Untersuchungen und den Nutzen, den man aus diesen für die Sprachvermittlung ziehen kann.

\section{Sprach(en)politik in Europa}

Im ersten Teil des Jahrbuchs geht es um Sprach- und Sprachenpolitik in Europa, ein Thema, das mehrere Betrachtungsperspektiven besitzt: Ist das Deutsche inzwischen eine „normale“ Sprache im Konzert der Sprachen Europas und wird es aus kulturellen, wirtschaftlichen und touristischen Gründen erlernt? Oder bringt die Tatsache, dass der deutschsprachige Raum mit seinen etwa 100 Millionen muttersprachlichen Sprecherinnen und Sprechern den größten europäischen Sprach- 
raum bildet, eine vielleicht ungewollte Dominanz mit sich? Wie wirkt sich dies auf die Sprachpolitik der Europäischen Union aus?

Einen Einstieg in die Diskussion dieser Fragen bietet Johannes Ebert, Generalsekretär des Goethe-Instituts, mit seinem Beitrag „Per Anhalter durch die Deutsch-Galaxis: Zur Situation der deutschen Sprache in Europa“. Das Deutsche befindet sich zwar aufgrund der Dominanz des Englischen und auch diverser Nationalisierungsbestrebungen in Europa unter erheblichem Druck, kann aber einen wachsenden Erfolg als zweite Fremdsprache nach dem Englischen verzeichnen. Auch der individuelle wirtschaftliche Nutzen des Erlernens der deutschen Sprache ist in letzter Zeit weiter in den Vordergrund getreten. Wie unterschiedlich sich jedoch die Situation darstellt, wenn man einzelne Länder genauer betrachtet, zeigt Ebert anhand von Polen, Frankreich und Russland. Das GoetheInstitut stellt sich auf die jeweiligen Umstände ein und unternimmt auch auf direktem Wege in den europäischen Institutionen den Versuch, die deutsche Sprache als Arbeitssprache durch ganz praktische Maßnahmen zu stärken.

Die Mechanismen, durch die Sprachenpolitik in einem Land Wirkung hervorruft, beleuchtet Vít Dovalil aus Prag. Er wendet die sogenannte Sprachmanagementtheorie an, um das Handeln verschiedener Akteure mit ihren divergierenden Interessen zu erfassen. Die Frage, die sich für Dovalil in seiner Untersuchung daraus ableitet, ist die nach dem Einfluss dieser Akteure im soziokulturellen Kontext des Deutschen in Europa. Er beantwortet sie mit Blick auf die Situation in Tschechien einerseits durch die Sprachenpolitik im schulischen Bereich und durch Beispiele grenzübergreifender Kooperationen zwischen Kommunen andererseits.

Rahel Beyer und Albrecht Plewnia vom IDS in Mannheim betrachten die besondere Situation der Mehrsprachigkeit und des Sprachkontakts in Grenzräumen. Sie erörtern die Spezifika der Grenzräume des deutschen Sprachraums zu Dänemark, Belgien, Italien und Frankreich und vertiefen ihre Darstellung exemplarisch anhand der laienlinguistischen Bewertung der Mehrsprachigkeit in OstLothringen. Sie führen dazu Daten der Zuordnung des lothringischen Dialekts als einer germanophonen Varietät auf, die in Interviews mit Sprecherinnen und Sprechern erhoben wurden. Die interessante Erkenntnis dabei liegt darin, dass eine solche Varietät als völlig losgelöst vom Deutschen angesehen werden kann, wenn das Hochdeutsche nicht als Dachsprache auf die sprachliche Situation in einer Region einwirkt.

Welchen Status hat das Deutsche als Studienfach in Europa? Mit dieser Frage befasst sich Marina Foschi Albert in ihrem Beitrag. Sie entfaltet Schritt für Schritt die Motivation, aufgrund derer Studierende in das „Ökosystem der Germanistik“ eintreten. Sie lenkt dabei den Blick auf die Internationalität der deutschen Sprache und die internationale Germanistik, die sich zu einer „EU-Germanistik“ entwickelt hat und sich dabei das germanistische Teilgebiet Deutsch als Fremdspra- 
che von vornherein in diesem europäischen Kontext entfalten kann. Die Autorin schließt den Bogen der vier Beiträge zum sprachenpolitischen Status der deutschen Sprache mit ihrer Frage nach der Nachhaltigkeit der internationalen Germanistik, die die Basis für den Status des Deutschen in Europa bildet.

\section{Erwerb, Konvergenzen, Divergenzen und Wandel des Deutschen im Europäischen Kontext}

Im zweiten Teil des Jahrbuchs geht es um grammatische, sprachsystematische Fragestellungen zum Deutschen und anderer europäischer Sprachen. Den Schwerpunkt bilden Themen wie Spracherwerb, Konvergenzen, Divergenzen und Wandel des Deutschen im Europäischen Kontext: Im Einzelnen werden folgende Fragen diskutiert: Wie gestaltet sich der Erwerb des Deutschen vor dem Hintergrund verschiedener Lerner-Profile? Mit welchen theoretischen Konzepten und Modellen lassen sich Konvergenzen und Divergenzen in Sprachen und Schriftsystemen erarbeiten? Welche methodischen Zugänge können als Desiderate für die kontrastive Forschung gelten? Welche Fortschritte sind zu verzeichnen? Zeigen Sprachen Europas systematische und vergleichbare Dynamiken des Wandels und erfasst der Wandel die Standardsprache?

Den Einstieg in das grammatische, sprachsystematische Thema legen Natalia Gagarina, Sophia Czapka, Nathalie Topaj und Manfred Krifka vom LeibnizZentrum Allgemeine Sprachwissenschaft zu „Erwerbsprofilen des Deutschen im mehrsprachigen Kontext“ vor. Jenseits des Standardszenarios eines muttersprachlichen Erwerbs in einem deutschsprachigen Land werden verschiedene Typen des Erwerbs des Deutschen als zweite Sprache festgestellt, die das Ergebnis von Langzeitstudien sind. Für Kinder mit russischer und türkischer Familiensprache, die das Deutsche als Zweitsprache erwerben, zeigen sich Abweichungen in den monolingualen Erwerbsverläufen. Im Kontext des mehrsprachigen Erwerbs zeigt sich, dass, für einen früheren L2-Erwerbsbeginn, ein reicher und nachhaltiger Input, wie durch explizite Sprachfördermaßnahmen, den Erwerb des Deutschen fördern und diese drei Punkte - früher Einstieg, reicher und nachhaltiger Input Prädiktoren der früheren Literalität bilden.

Nanna Fuhrhop von der Universität Oldenburg skizziert ein theoretisches Konzept für die Begründung einer „Vergleichenden Graphematik“ in Alphabet basierten Schriftsystemen. Konvergenzen und Divergenzen bei der Untersuchung von vier Schriftsystemen (Deutsch, Englisch, Niederländisch und Französisch) und sechs graphematischen Phänomene, unter ihnen Doppelkonsonanten- und Apostrophschreibung, werden bezüglich ihrer Kontextbedingungen quantitativ- 
explorativ erhoben und qualitativ ausgewertet. Auf dieser Grundlage werden Parameter zur Beschreibung der Phänomene entwickelt, wobei jedes spezifische Schriftsystem zur Aufdeckung übergeordneter Prinzipien einer allgemeinen Graphematik beitragen kann.

Um Konvergenzen und Divergenzen im Sprachsystem geht es im Beitrag von Lutz Gunkel und Jutta M. Hartmann vom IDS Mannheim und der Universität Bielefeld. Im Beitrag „Präpositionalobjektsätze im europäischen Vergleich“ (PO) werden drei ausgewählte germanische Sprachen (Schwedisch, Niederländisch sowie Deutsch) und zwei romanische Sprachen (Französisch und Italienisch) sprachvergleichend untersucht. Es werden zwei Strategien der Anbindung von Präpositionalobjektsätzen (PO) an den Trägersatz beobachtet: eine direkte (für Schwedisch als nordgermanische Sprache und die untersuchten romanischen Sprachen) und eine indirekte (Deutsch und Niederländisch) über die Anbindung mit einer komplexen Proform, die beim Deutschen gleichzeitig eine komplexe Konstituente mit dem PO-Objekt bilden kann. Des Weiteren weisen verschiedene Tests darauf hin, dass in allen untersuchten Sprachen immer ein formales „Präpositionselement“ syntaktisch präsent sein muss - lexikalisch realisiert oder unrealisiert.

Ob Sprachen Europas systematische und vergleichbare Dynamiken des Wandels zeigen und wie der Wandel zu identifizieren ist, zeigen Thomas Stolz und Nataliya Levkovych von der Universität Bremen in ihrem Beitrag „Über die Diffusion von /y/ in Europa und wie viel Verantwortung das Deutsche dafür trägt“. Den Untersuchungsrahmen bildet das Projekt Phonologischer Atlas Europas (Phon@Europe). Anhand der geografischen Distribution des hohen vorderen gerundeten Vokalphonems /y/ in Europa wird u. a. diskutiert, welche Rolle der Sprachkontakt - insbesondere mit dem Deutschen - bei der Verbreitung von /y/ gespielt haben könnte oder ob und wo von einer kontaktunabhängigen Entstehung von /y/ ausgegangen werden kann. Die Befunde werden kontakt- und areallinguistisch ausgewertet und das Deutsche in der phonologischen Landschaft Europas situiert.

Im Beitrag von Peter Auer von der Universität Freiburg „Gibt es einen deutschen Neo-Standard und - wenn ja - wie verhält er sich zu den Entwicklungen der Standards anderer europäischer Sprachen?“ wird untersucht, ob Wandel auch die Standardsprache erfasst. Es wird die Hypothese eines neuen Standards (Neo-Standard) als ,beste Sprache، vertreten und diese Auffassung hinsichtlich zweier Gegenargumente verteidigt, a) dass es sich um de-standardisierte Sprechweisen handelt und b) dass es sich bei Neo-Standard um einfachen Sprachwandel innerhalb des weiter existierenden einzigen Standards handelt, der von manchen Sprecherinnen und Sprechern mehr realisiert wird als von anderen. Die Untersuchung nimmt eine soziolinguistische, vergleichende Perspektive ein, die auch die Entwicklung des Standards in anderen europäischen Ländern berücksichtigt, zu deren Neo-Standards bereits umfangreich geforscht worden ist. 


\section{Methoden - Sprachressourcen und Infrastrukturen}

Der dritte Teil des Jahrbuchs behandelt methodische Fragen, wobei hier Methoden der Digitalen Sprachwissenschaft, insbesondere beim Umgang mit digitalen Sprachdaten, betrachtet werden. Zudem werden Aspekte von Forschungsinfrastrukturen besprochen. Im Zentrum stehen Sprachressourcen für das Deutsche, die für sprachvergleichende Untersuchungen genutzt werden können. Dieser Teil umfasst drei Beiträge, die komplementär wichtige Aspekte dieser digitalen Methoden ausleuchten.

Erhard Hinrichs’ (IDS/Tübingen) Beitrag „Multilinguale Sprachressourcen für die linguistische Forschung " beschreibt wichtige Sprachressourcen für das Deutsche. Einen Typ dieser Sprachdatensammlungen bilden Textkorpora. Dargestellt werden ihre Nutzungsmöglichkeiten für sprachvergleichende Analysen, wobei eine wichtige Vergleichssprache in diesem Beitrag das Englische ist. Die Wahl des Englischen liegt u. a. darin begründet, dass der Sprachvergleich die Verfügbarkeit von digitalen Sprachressourcen in der Vergleichssprache voraussetzt. Die anglistische Linguistik hat durch ihre frühe Nutzung von Sprachkorpora in diesem Gebiet eine besondere Bedeutung. Hierdurch - und durch den sehr großen Sprachraum des Englischen - sind heute englischsprachige Ressourcen in großem Umfang verfügbar, die als Vergleichskorpora genutzt werden können. Nach der Beschreibung der Textkorpora stellt der Artikel das Konzept der Baumbanken vor, worunter die syntaktisch annotierten Textkorpora verstanden werden. Die damit einhergehende größere Komplexität von Korpora wird durch die Darstellung von verschiedenen, syntaktischen Ansätzen - insbesondere von dependenzgrammatischen und konstituentenbasierten Formaten - veranschaulicht. Der Sprachvergleich wird anschließend durch die Vorstellung mehrsprachiger Baumbanken, bei denen kompatible Annotationsformate gewählt werden, nochmals hervorgehoben. Der Beitrag schließt mit einer Beschreibung der Forschungsdateninfrastruktur CLARIN, die ein europäisches Dach in Form einer Organisationsstruktur, dem CLARIN ERIC, besitzt. Unter diesem Dach wurden in den vergangenen Jahren viele nationale Forschungsinfrastrukturen gegründet. Ein besonderer Schwerpunkt in der Darstellung bildet die deutsche Forschungsinfrastruktur CLARIN-D.

Der Beitrag „Von monolingualen Korpora über Parallel- und Vergleichskorpora zum Europäischen Referenzkorpus EuReCo“ von Beata Trawiński und Marc Kupietz (beide IDS) zeigt einen Weg, der von nationalen, einzelsprachlichen Korpora zu einem großen, multilingualen Korpus-Netzwerk, das in den kommenden Jahren kontinuierlich weiter ausgebaut werden soll, führt. Orientiert an der 
Namensgebung des Deutschen Referenzkorpus DEREKo wurde die beschriebene Initiative zum Aufbau eines europäischen Referenzkorpus EuReCo getauft. Der Beitrag beschreibt die Nutzungsmöglichkeit mehrsprachiger Korpora sowie ihre methodischen Ausdifferenzierungen in einerseits parallelen und andererseits vergleichbaren Korpora. Im Verlauf des Beitrages werden die Korpora DruKoLA, eine deutsch-rumänische Korpussammlung, und DeutUng, eine deutsch-ungarische Korpusinitiative, beschrieben. Da für einen korpusbasierten Sprachvergleich, genau wie für die Nutzung einsprachiger Korpora, digitale Analysesysteme genutzt werden müssen, wird im abschließenden Teil die Anpassung der am IDS entwickelten Korpusanalyse-Plattform KorAP für die Nutzung vergleichbarer Korpora beschrieben, durch die die Verwendung der deutsch-rumänischen bzw. der deutsch-ungarischen Korpusressourcen mit einem vereinheitlichten Zugang ermöglicht wird.

Der Beitrag „Tiefe Analyse von Lernerkorpora“ von Anke Lüdeling, Hagen Hirschmann, Anna Shadrova und Shujun Wan (alle Humboldt-Universität Berlin) behandelt den Korpustyp Lernerkorpora und ihre Nutzungsmöglichkeiten. Diese Textsammlungen haben in der jüngeren Vergangenheit eine immer größere Bedeutung erhalten. Gerade für das Tagungsthema - Deutsch in Europa - ist die Beschreibung von Lernerkorpora hochrelevant, da das Deutsche - wie oben erwähnt - nach wie vor eine bedeutende Fremdsprache in vielen europäischen Ländern bildet. Lernerkorpora stellen ganz neue Herausforderungen an die Aufbereitung und Analyse von Korpusressourcen, da Lernerinnen und Lerner nicht fehlerfrei schreiben. Die Analyse der Fehler von Lernenden des Deutschen hilft insbesondere auch der Forschung im Bereich „Deutsch als Fremdsprache“, bietet aber darüber hinaus auch einen Einblick in die allgemeinen Aspekte der menschlichen Sprachverarbeitung, da Fehler häufig von der Erstsprache beeinflusst werden. Die in dem Beitrag dargestellten Methoden gehen über die klassische Analyse von Lernerkorpora weit hinaus, da sie eine Tiefenanalyse vorschlagen und beschreiben.

\section{Methodenmesse}

Die traditionelle Methodenmesse der IDS-Jahrestagung mit Posterbeiträgen und Systemdemonstrationen wurde in diesem Jahr von Harald Lüngen organisiert. Auf ihr wurden gemäß dem Tagungsmotto aktuelle Korpora, Ressourcen und Methoden für die kontrastive Forschung über das Deutsche im Vergleich mit anderen europäischen Sprachen vorgestellt. Der vorliegende Band enthält sieben Kurzbeiträge zu diesen Präsentationen. 
Nils Diewald, Franck Bodmer, Peter Harders, Elena Irimia, Marc Kupietz, Eliza Margaretha und Helge Stallkamp berichten darüber, wie in der EuReCoInitiative mittels KorAP, der Korpusrechercheplattform des IDS, anhand harmonisierter Metadaten europäischer Referenzkorpora virtuelle vergleichbare Teilkorpora definiert und kontrastiv analysiert werden. Aus der Abteilung Grammatik des IDS gibt es je einen Beitrag über neue Entwicklungen des bewährten Grammatikinformationssystems grammis (Stefan Falke, Saskia Ripp, Roman Schneider, Ulrich Hermann Waßner) sowie zu der neuen Ressource CoMParS mit annotierten multilingualen Parallelsequenzen des Deutschen und anderer europäischer Sprachen (Beata Trawiński, Susan Schlotthauer, Piotr Bański). Tomáš Káňa (Brno) beschreibt Inhalte und Einsatz des klassischen vergleichbaren Korpus InterCorp mit Textdaten aus vierzig vorwiegend europäischen Sprachen. Andreas Nolda, Adrien Barbaresi und Alexander Geyken (Berlin) berichten über das neue, sehr große ZDL-Regionalkorpus für areale Untersuchungen des Deutschen und Erhard Hinrichs, Patricia Fischer, Yana Strakatova über Rover und TüNDRA, die Tübinger Visualisierungsplattformen für Wortnetze und Baumbanken. Schließlich stellen Antonina Werthmann, Andreas Witt und Jutta Bopp (IDS) CLARIAH-DE vor, das Verbundprojekt zur Weiterentwicklung der nationalen Forschungsinfrastrukturen für die Geisteswissenschaften und verwandter Disziplinen und Teil des oben genannten Netzwerks CLARIN ERIC, zu dem mehr als zwanzig europäische Forschungsinfrastrukturkonsortien gehören. 
